\title{
The shift from low to high non-structural protein 1 expression in rotavirus-infected MA-104 cells
}

\author{
Laura Martínez-Álvarez, Carolina Piña-Vázquez, Wilbert Zarco, Luis Padilla-Noriega/+ \\ ${ }^{1}$ Instituto de Investigaciones Biomédicas, Universidad Nacional Autónoma de México, Mexico DF, Mexico
}

\begin{abstract}
A hallmark of group/species A rotavirus (RVA) replication in MA-104 cells is the logarithmic increase in viral $m R N A s$ that occurs four-12 h post-infection. Viral protein synthesis typically lags closely behind $m R N A$ synthesis but continues after mRNA levels plateau. However, RVA non-structural protein 1 (NSPI) is present at very low levels throughout viral replication despite showing robust protein synthesis. NSPI has the contrasting properties of being susceptible to proteasomal degradation, but being stabilised against proteasomal degradation by viral proteins and/ or viral mRNAs. We aimed to determine the kinetics of the accumulation and intracellular distribution of NSP1 in MA-104 cells infected with rhesus rotavirus (RRV). NSP1 preferentially localises to the perinuclear region of the cytoplasm of infected cells, forming abundant granules that are heterogeneous in size. Late in infection, large NSP1 granules predominate, coincident with a shift from low to high NSP1 expression levels. Our results indicate that rotavirus NSPI is a late viral protein in MA-104 cells infected with RRV, presumably as a result of altered protein turnover.
\end{abstract}

Key words: rotavirus - gene expression - interferons type 1 - proteasome endopeptidase complex - ubiquitin protein ligases

Group/species A rotaviruses (RVAs) belong to the Reoviridae family and are one of the major causes of gastroenteritis in infants and young children (Anonymous 2008). The viral particle consists of three concentric protein layers that enclose a genome of 11 segments of double-stranded RNA (dsRNA). The viral genome encodes six structural proteins [viral protein (VP) VP1-VP4, VP6, VP7] and five or six non-structural proteins [(NSP)1-NSP5 and, in some strains, NSP6]. Of the structural proteins, VP4 and VP7 form the outer layer, VP6 the intermediate layer and VP2 the inner layer. The viral RNA polymerase VP1 and the RNA-capping enzyme VP3 are associated with the inner layer and the viral genome (Estes \& Kapikian 2007).

RVA entry into the host cell is accompanied by loss of the outer layer, converting the triple-layered particles (TLPs) into double-layered particles (DLPs), which are transcriptionally active. Viral mRNAs are capped, but not polyadenylated, and they have the dual roles of being translated into viral proteins and being templates for the production of genomic dsRNA. The putative sites of genome transcription, replication and DLP assembly are the viroplasms, which are cytoplasmic inclusion bodies made of viral RNAs and proteins. In MA-104, a vervet monkey kidney cell line, the DLPs assemble in the viroplasms and bud through the endoplasmic reticulum (ER), thus acquiring a transient lipid envelope. In the ER, the envelope is lost concurrently with the assembly of the outermost layer to produce TLPs and the mature virions are released by cell lysis (Estes \& Kapikian 2007).

doi: 10.1590/0074-0276108042013005

Financial support: CONACyT (51029-Z and 168046), UNAM

(DGAPA-PAPIIT-UNAM IN224809 and IN201212)

+ Corresponding author: 1padilla@unam.mx

Received 2 October 2012

Accepted 14 February 2013
The kinetics of viral mRNA synthesis in MA-104 cells infected with rhesus rotavirus (RRV) consist of a slight linear increase during the first $4 \mathrm{~h}$ post-infection (hpi) followed by a logarithmic increase from 4-12 hpi (Ayala-Breton et al. 2009). Protein synthesis lags closely behind mRNA synthesis, such that typical RVA proteins, such as NSP2 (the major component of viroplasms), start to accumulate sharply at $4 \mathrm{hpi}$ and reach a very high level at 8 hpi (Carreno-Torres et al. 2010). The kinetics of dsRNA and infectious virus progeny production show a 2-h delay compared to mRNA synthesis, such that a slight linear increase occurs from 3-6 hpi followed by a logarithmic increase from 6-12 hpi (Ayala-Breton et al. 2009). Moreover, infectious virus production reaches its maximum level at $12 \mathrm{hpi}$ (Estes et al. 1979, McCrae \& Faulkner-Valle 1981, Johnson \& McCrae 1989, AyalaBreton et al. 2009).

NSP1 is a 53-kDa RNA-binding protein that localises to the cytoplasm of infected cells (Hua \& Patton 1994, Hua et al. 1994). Although it is the most variable viral protein, NSP1 contains a conserved set of seven cysteines and one histidine near the N-terminus (residues 44-74 of RRV gene 5), which has been proposed to be a really interesting new gene (RING) domain, which is characteristic of E3 ubiquitin-protein ligases (Graff et al. 2007, Pina-Vazquez et al. 2007). NSP1 interacts with interferon (IFN) regulatory factors (IRFs) 3,5 and 7 and the $\beta$-transducin repeat-containing protein $(\beta-\operatorname{TrCP})$, the component of the $\mathrm{E} 3$ ubiquitin ligase complex responsible for activating NF- $\kappa$ B. This interaction induces the degradation of IRFs and $\beta$-TrCP through the proteasome, resulting in suppression of the type I IFN response (Arnold \& Patton 2010).

In contrast to the high expression levels of typical RVA proteins, such as NSP2 and VP6, NSP1 has a low expression level in RVA-infected cells and is barely detectable by metabolic labelling (Ericson et al. 1982, Johnson \& McCrae 1989, Pina-Vazquez et al. 2007). The 
determinants of the low NSP1 expression level have not been thoroughly studied. Polyribosome analysis indicated that NSP1 mRNA has a lower translational efficiency than VP6 mRNA in MA-104 cells infected with the RVA strain B641 (Mitzel et al. 2003). Using a highly efficient vaccinia virus expression system, we previously found that despite having robust protein synthesis, RRV NSP1 has very low expression levels due to its high susceptibility to proteasomal degradation. Moreover, this proteasomal degradation was prevented by transfection with the full complement of translation-competent viral mRNAs, thus suggesting that viral proteins alone or in combination with viral mRNAs prevent the degradation of NSP1 by the proteasome (Pina-Vazquez et al. 2007). Because RING-domain E3 ubiquitin ligases are capable of self-ubiquitination in the absence of their protein targets, the proposed role of NSP1 as an E3 ubiquitin ligase would allow this protein to auto-regulate its expression level via self-ubiquitination and degradation by the proteasome. However, in the presence of viral or cellular factors that are able to bind NSP1, self-ubiquitination would be prevented, thus upregulating its expression (Graff et al. 2007, Pina-Vazquez et al. 2007).

Because a detailed study of NSP1 expression throughout RVA infection is lacking, we determined the intracellular distribution and expression kinetics of NSP1 in RRV-infected cells using immunofluorescence (IF) and Western blot (WB) analyses, respectively.

\section{MATERIALS AND METHODS}

Cell lines and viruses - RRV was obtained from $\mathrm{HB}$ Greenberg (Stanford University, Stanford, CA). The vaccinia virus vT7lacOI was obtained from B Moss (National Institutes of Health, Bethesda, MD). The recombinant vaccinia virus vNSP1, which was used for expressing NSP1 from RRV, was previously produced in our laboratory (Pina-Vazquez et al. 2007). NSP1 expression in BSC-1 cells was based on the vaccinia virus/lac operon/ T7 RNA polymerase/encephalomyocarditis (EMC) internal ribosome entry site (IRES) (VOTE) mammalian cell system developed by B Moss (Ward et al. 1995). In this system, the cells are infected with a recombinant vaccinia virus carrying the target gene, which is transcribed from a T7 RNA polymerase promoter regulated by an Escherichia coli lac operator that is produced at high levels due to the IRES of the EMC virus. The vervet monkey kidney cell lines MA-104 and BSC-1 were obtained from R Wyatt (National Institutes of Health, Bethesda, MD) and R Rosales (Universidad Nacional Autónoma de México, Mexico City), respectively. The human cervix adenocarcinoma cell line HeLa was obtained from A González (Universidad Nacional Autónoma de México, Mexico City). RRV was propagated in MA-104 cells (Kaljot et al. 1988) and vaccinia viruses were replicated in adherent HeLa cells (Earl et al. 2007).

Antibodies - The anti-NSP2 guinea pig serum was produced in our laboratory as previously described (Cabral-Romero \& Padilla-Noriega 2006). The monoclonal antibodies (Mabs) from the ascitic fluid used to detect the RVA proteins were obtained from HB Green- berg: 3A8/6E8 anti-VP2 (Svensson et al. 1990), 1E11 anti-VP6 (Coria-Galindo et al. 2009), 4F8 anti-VP7 (Shaw et al. 1985) and 191 anti-NSP2 (Bass et al. 1990).

For this study, we produced 12 sera that were hyperimmune to a C-terminal peptide of RRV NSP1 or full-length recombinant NSP1 from RRV. Here, we describe only the production of serum that was hyperimmune to full-length NSP1 and showed the best signal/noise ratio with IF of RRV-infected cells; this serum was used throughout the study. Gene 5 of RRV, which codes for NSP1, was amplified from the viral genomic dsRNA with the ThermoScript reverse transcription-polymerase chain reaction system (Invitrogen, Carlsbad, CA) and cloned into the NcoI-XhoI sites of the plasmid pET28b (Novagen, Madison, WI) to produce $\mathrm{pET} 28 \mathrm{~b}-\mathrm{NSP}$. The following specific primers were used to amplify the NSP1 gene (GenBank: U08433): 5'-CAATATTCCATGGCAACCTTTAAG-3' and 5'-GATAACTCGAGGTCGAGGTCGTCATCTTCAGAG-3'. A hexahistidine tail was added to the $\mathrm{C}$-terminus in this construct. The insert was sequenced from both strands.

NSP1 expression was induced in E. coli BL21-DE3 transformed with pET28b-NSP1 with $1 \mathrm{mM}$ isopropylbeta-d-thiogalactopyranoside (IPTG) for $6 \mathrm{~h}$ at $37^{\circ} \mathrm{C}$, starting at an $\mathrm{OD}_{600}$ of 0.8 . The bacterial cells were disrupted by sonication in lysis buffer [phosphate-buffered saline (PBS)] without calcium or magnesium plus an ethylenediamine tetraacetic acid-free protease inhibitor cocktail (Roche, Mannheim, Germany). The quantity of NSP1 in the insoluble fraction was determined using sodium dodecyl sulphate-polyacrylamide gel electrophoresis (SDS-PAGE) (Laemmli 1970). To obtain the NSP1 antigen, the band corresponding to NSP1 was excised from the gel and pulverised at $-70^{\circ} \mathrm{C}$. The NSP1 antigen was administered intramuscularly and intraperitoneally to guinea pigs in four $100-\mu \mathrm{g}$ doses with complete Freund's adjuvant (Sigma-Aldrich, Saint Louis, MI) for the first dose and incomplete adjuvant for subsequent doses. The guinea pigs were bled 10 days after the last dose and the serum was separated.

Infection of MA-104 cells with RRV - The viral inoculum was activated by treatment with $10 \mu \mathrm{g} / \mathrm{mL}$ trypsin (1:250, Invitrogen) for $30 \mathrm{~min}$ at $37^{\circ} \mathrm{C}$. Confluent MA104 monolayers were infected with activated RRV in serum-free minimum essential medium (MEM) (Invitrogen) at the indicated multiplicity of infection (MOI). At $1 \mathrm{~h}$ after infection, the viral inoculum was replaced with serum-free MEM and the cells were incubated at $37^{\circ} \mathrm{C}$ until the indicated time pi. In some experiments, the RRV infections were synchronised by treatment with the 4F8 Mab at $1 \mathrm{hpi}$, thus neutralising both adsorbed and non-adsorbed virions as previously described (Ruggeri \& Greenberg 1991). Briefly, the cells were washed with serum-free MEM at $4^{\circ} \mathrm{C}$ and incubated with the neutralising Mab 4F8 diluted 1:200 in serum-free MEM at $4^{\circ} \mathrm{C}$. After $1 \mathrm{~h}$, the cells were washed with serum-free MEM and incubated at $37^{\circ} \mathrm{C}$ until the indicated time pi.

Infection of BSC-1 cells with vaccinia viruses BSC-1 cells were grown in 16-well chambered slides (Nalge Nunc, Rochester, NY) at $37^{\circ} \mathrm{C}$. Prior to infection, the viral inoculum was activated with $125 \mu \mathrm{g} / \mathrm{mL}$ of 
trypsin (1:250, Invitrogen) for $30 \mathrm{~min}$ at $37^{\circ} \mathrm{C}$ and further homogenised in a cup sonicator for $1 \mathrm{~min}$ as previously described (Elroy-Stein \& Moss 2007). The BSC-1 monolayers were infected with activated vNSP1 or vT7lacOI at the indicated MOI, the inoculum was replaced at $1 \mathrm{hpi}$ with MEM $+2.5 \% \mathrm{FBS}+1 \mathrm{mM}$ IPTG, the cells were incubated at $37^{\circ} \mathrm{C}$ and further processed for IF at the indicated times pi.

$W B S$ - MA-104 cells grown in 12-well plates were mock-infected or infected with RRV as described above. At the indicated times pi, the cells were harvested as previously described (Padilla-Noriega et al. 2002). The cell pellets obtained were resuspended in $20 \mu \mathrm{L}$ of lysis buffer [50 mM Tris- $\mathrm{HCl}$, pH 7.5, 5 mM MgCl, $0.4 \mathrm{U} / \mathrm{mL}$ DNase I, $0.01 \mathrm{mg} / \mathrm{mL}$ RNase A and $37 \mathrm{mg} / \mathrm{mL}$ of protease inhibitor cocktail (P8340, Sigma-Aldrich)] and disrupted in a cup sonicator for $1 \mathrm{~min}$ at $4^{\circ} \mathrm{C}$. The lysed cells were mixed with $20 \mu \mathrm{L}$ of Laemmli sample buffer and boiled for $5 \mathrm{~min}$. One half of the cell harvests from each well were resolved on a $12 \%$ SDS-PAGE gel and then transferred to polyvinylidene fluoride membranes (162-0177, BioRad, Hercules, CA). The membranes were blocked with $5 \%$ non-fat dry milk in Tris-buffered saline $+0.15 \%$ Tween 20 (TBS-Tween) at room temperature (RT) and further incubated for $2.5 \mathrm{~h}$ at RT with anti-NSP1 (1:500 or 1:5,000) or $1 \mathrm{~h}$ with anti-glyceraldehyde-3-phosphate dehydrogenase (GAPDH) (MAB374, Millipore, Billerica, MA) (1:10,000), both of which were diluted with $1 \%$ non-fat dry milk in TBS-Tween. The blots were then incubated for $1 \mathrm{~h}$ with appropriate horseradish peroxidase-conjugated anti-IgG antibodies diluted with $1 \%$ non-fat dry milk in TBS-Tween. Finally, the blots were developed with SuperSignal West Femto chemiluminescence substrate (Pierce, Rockford, IL) according to the manufacturer's instructions. The blots were scanned and quantitated using Image J $1.43 \mathrm{u}$ software.

IF microscopy - Confluent MA-104 cell monolayers in 16-well chambered slides were infected as described above and at the indicated times pi, the cells were washed with cold PBS and fixed for 15 min at RT with 4\% paraformaldehyde in PBS. The cells were then permeabilised with PBS $+0.1 \%$ Triton X-100 (PBST) for $10 \mathrm{~min}$ and blocked for $1 \mathrm{~h}$ with PBST $+1 \%$ bovine serum albumin (BSA) at RT. The cells were then incubated for $1 \mathrm{~h}$ with one of the following primary antibodies diluted in PBST $+1 \%$ BSA: anti-NSP1 serum (1:200), anti-NSP2 Mab (1:40,000), anti-VP6 Mab (1:40,000) or anti-VP2 Mab $(1: 50,000)$. After three washes with cold PBST $+1 \%$ BSA, the cells were incubated for $1 \mathrm{~h}$ with Alexa Fluor 488 or Alexa Fluor 594 secondary antibodies (Invitrogen) diluted in PBST $+1 \%$ BSA. The cells were washed three times with cold PBST and the nuclei were stained with $0.1 \mu \mathrm{g} / \mathrm{mL}$ 4',6-diamino-2-phenylindole (DAPI) (Sigma-Aldrich). The cover slips were mounted in Dako mounting medium (Dako Corporation, Carpinteria, CA) and analysed using epifluorescence microscopy. Images were acquired using Image-Pro Plus v6.2 software (Media Cybernetics, Bethesda, MD) with an Olympus IX70 microscope coupled to a CoolSNAP-Pro camera (Media Cybernetics) and analysed using ImageJ.

\section{RESULTS}

Recombinant NSP1 expressed in a vaccinia system localises to the cytoplasm with a diffuse and granular appearance - To determine the intracellular distribution of NSP1 in the absence of other viral proteins and/or viral RNAs, we infected the vervet monkey kidney cell line BSC-1 with the recombinant vaccinia virus vNSP1, which had previously been developed to express RRV NSP1 (Pina-Vazquez et al. 2007). As a negative control, cells were infected with vT7lacOI, the parental vaccinia virus used to produce vNSP1 (Ward et al. 1995). At 15 hpi, the cell monolayers were analysed using IF with a guinea pig anti-NSP1 serum. No NSP1 signal was detected in the control cells infected with vT7lacOI (Fig. 1A, left image). In contrast, recombinant NSP1 was detected in the cytoplasm of cells infected with vNSP1. NSP1 displayed a diffuse distribution pattern and a few granules throughout the cytoplasm (Fig. 1A, right image).

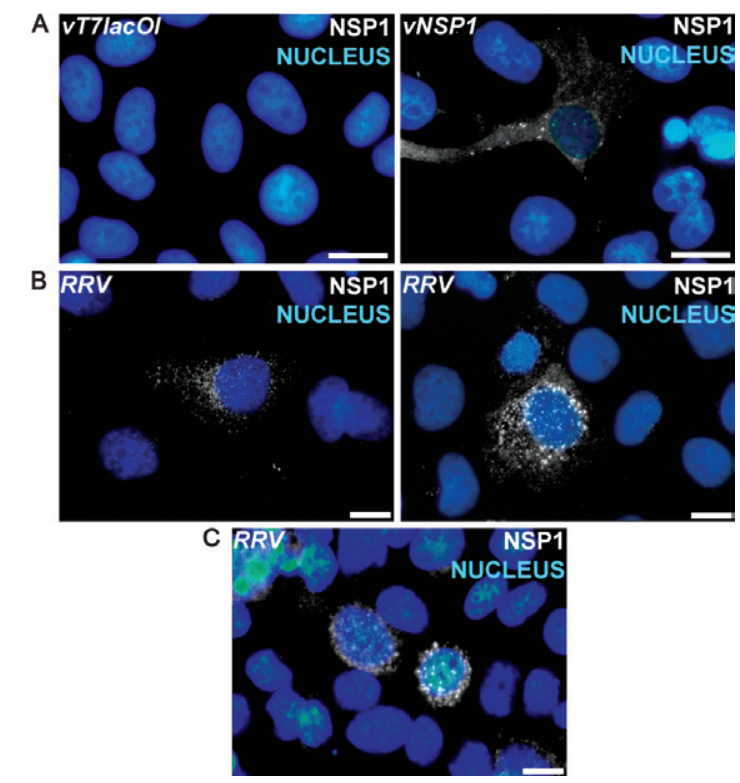

Fig. 1: intracellular localisation of rhesus rotavirus (RRV) nonstructural protein 1 (NSP1). A: recombinant NSP1 localises to the cytoplasm with a diffuse and granular appearance. BSC-1 cell monolayers were infected with the vaccinia virus vNSP1 or vT7lacOI at a multiplicity of infection (MOI) of 0.1 and induced with $1 \mathrm{mM}$ isopropyl-beta-d-thiogalactopyranoside. At $15 \mathrm{~h}$ post-infection (hpi), the cells were processed using immunofluorescence (IF) with anti-NSP1 (white) and 4',6-diamino-2-phenylindole (DAPI) (blue). NSP1 was not detected in the cells infected with the parental vaccinia vT7lacOI (left picture), while in cells infected with vNSP1, a diffuse and granular appearance of NSP1 was observed (right picture); B: NSP1 localises to the cytoplasm in RRV-infected cells, with preferential localisation to the perinuclear region and granular appearance. MA-104 cell monolayers were infected with RRV at an MOI of 0.1 (upper row) or 1 (lower row). At $12 \mathrm{hpi}$, the cells were processed using IF with anti-NSP1 (white) and DAPI (blue). NSP1 localised preferentially to the perinuclear region, with some cells showing predominantly small granules (upper row, left picture) and others large granules (upper row, right picture). The extent of localisation differences and the heterogeneity in the levels of NSP1 expression in different cells are shown using a higher MOI (lower picture). Bar $=10 \mu$. 
NSP1 shows a granular appearance and localises preferentially in the perinuclear region of the cytoplasm of RRV-infected cells - To determine the intracellular distribution of NSP1 in the context of viral infection, we infected MA-104 cells with RRV and analysed the cell monolayers using IF at 12 hpi. NSP1 localised to the cytoplasm, forming abundant granules with varied sizes ranging from small to large (Fig. 1B). We also observed a range of NSP1 expression levels that correlated with granule size; in cells with low expression, small granules predominated (Fig. 1B, upper left image), whereas in cells with high expression, large granules predominated (Fig. 1B, upper right image). Heterogeneity in NSP1 expression levels and granule sizes was observed at a lower magnification (Fig. 1B, lower image). Remarkably, NSP1 preferentially localised to the perinuclear region of RRV-infected cells. In contrast, the recombinant protein expressed with the vaccinia system was distributed throughout the cytoplasm, suggesting that perinuclear localisation is determined by viral factors.

Large NSP1 granules are detected only at late times $p i$ - Having established that NSP1 granules of different sizes can be detected using IF in RRV-infected cells, we investigated the kinetics of granule appearance. We infected MA-104 cells with RRV and analysed the distribution of NSP1 at different times pi using IF with anti-NSP1 serum. As an infection control, we performed double IF with the anti-NSP2 Mab to detect viroplasms. The distribution kinetics of NSP1 were assessed at each time pi by classifying a minimum of 50 rotavirus-infected cells according to the level of NSP1 expression (low or high) as determined by direct observation at the microscopic level (Fig. 1B). NSP1 expression was undetectable at 12 hpi in mock-infected cells (Fig. 2A), while NSP1 was detected at low levels from 6-9 hpi in RRVinfected cells (Fig. 2B, C). The intracellular distribution of NSP1 at 6 hpi and 9 hpi was granular and cytoplasmic, but showed no large granules. From 12 hpi until the final time point at $18 \mathrm{hpi}$, approximately $30-40 \%$ of infected cells displayed high NSP1 expression levels characterised by a confluence of large NSP1 granules (Fig. 2D-F). Two fields per row are shown in Fig. 2 to demonstrate the heterogeneity in the distribution and expression levels of NSP1 in infected cells under each condition. These results suggest that the formation of large NSP1 granules correlates with high expression levels.

NSP1 has a low expression level that increases at late times pi - Because our IF assays of NSP1 were not quantitative, we determined the kinetics of NSP1 expression in RRV-infected cells using WB analysis. Our hyperimmune anti-NSP1 serum detected a specific NSP1 band that migrated according to its expected molecular weight (Fig. 3A, Lane 2) and an intense non-specific cellular band in both mock-infected and RRV-infected cells (Fig. 3A, Lanes 1,2). The reactivity of our hyperimmune anti-NSP1 serum with a cellular protein did not preclude its use in WB analysis to determine the level of NSP1 expression because of the different electrophoretic migration of NSP1 and the cellular protein. We infected MA-104 cells with RRV at an MOI of 10 and measured the levels of NSP1 expression at different times pi (Fig. 3B). As controls, cellular GAPDH and viral NSP2 were also detected (Fig. 3B). Our results demonstrated that NSP1 had a low expression level at 6 hpi that increased by seven-fold at 12 hpi and remained similarly high until 18 hpi (Fig. 3C). The kinetics of NSP1 expression were different from the major viroplasm component NSP2, which was previously analysed using the same method, cell line, virus and MOI (Carreno-Torres et al. 2010). Carreno-Torres et al. (2010) found a sharp increase in the level of NSP2 expression starting at 4 hpi that correlated with the logarithmic phase of viral replication and increased viroplasm number. In contrast, we found a rise in NSP1 expression starting much later than NSP2 at 9 hpi. Based on the difference in the kinetics of accumulation of NSP1 vs. other viral proteins, such as NSP2, NSP1 is a late viral protein.

High levels of NSP1 expression in RRV-infected cells correlate with altered viroplasm morphology - While performing studies on the intracellular localisation of NSP1 in RRV-infected cells, we observed that cells with high NSP1 expression levels exhibited alterations in the morphology of the viroplasms detected with anti-NSP2 antibodies (Fig. 2). In RRV-infected MA-104 cells with low NSP1 expression levels, the intracellular distribution of NSP2 was characterised by small perinuclear

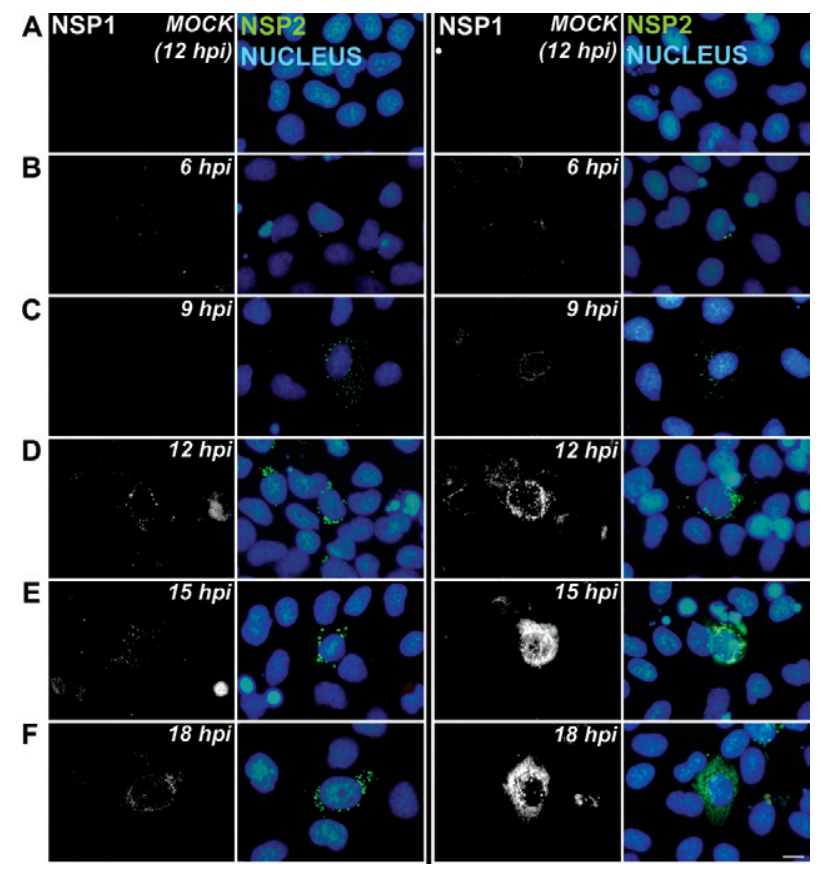

Fig. 2: large non-structural protein 1 (NSP1) granules are detected only late in infection in rhesus rotavirus (RRV)-infected cells. MA-104 cells were mock-infected (row A) or infected with RRV at a multiplicity of infection of 0.1 (rows B-F) and at different times post-infection (pi) (as indicated), the cells were processed using immunofluorescence with anti-NSP1 (white) or anti-NSP2 (green) and 4',6-diamino-2-phenylindole (blue). Two images are shown for each pi time, which illustrate the heterogeneity in the intensity of NSP1 fluorescence. hpi: hours pi. Bar $=10 \mu$. 
granules and the viroplasms, which are characteristically large, round structures that do not colocalise with NSP1, appeared normal (Fig. 2D-F, left panel). In contrast, cells with high NSP1 expression levels had altered viroplasm morphology characterised by a confluence of
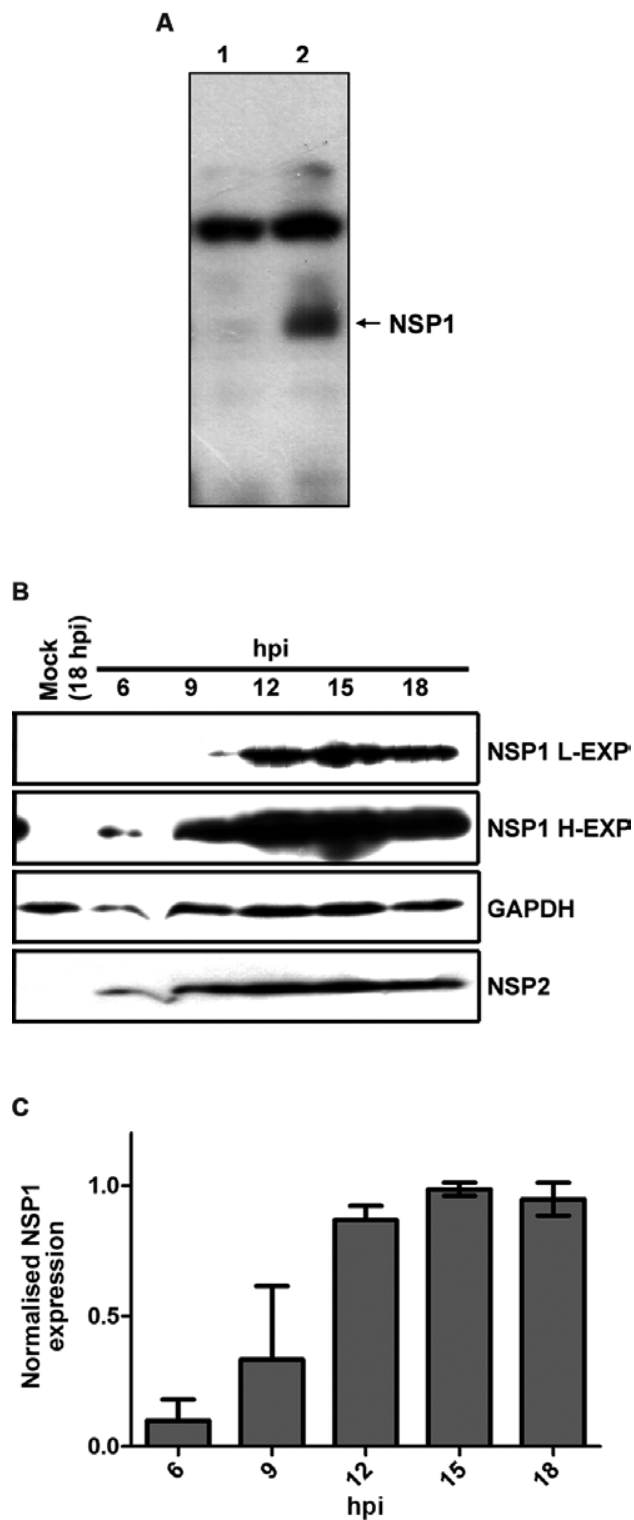

Fig. 3: non-structural protein 1 (NSP1) has a low expression level that increases late in infection in rhesus rotavirus (RRV)-infected cells. MA-104 cells were mock-infected or infected with RRV at a multiplicity of infection of 10 and the cells were processed using Western blot analysis with anti-NSP1, anti-NSP2 and anti- glyceraldehyde-3phosphate dehydrogenase (GAPDH) at different times post-infection (pi) (as indicated). The anti-NSP1 serum detected an intense cellular band in mock-infected cells (Lane 1, panel A), whereas a specific NSP1 band was detected only in RRV-infected cells (A). The results of one representative WB assay are shown (B), with two lengths of film exposure [low (NSP1 L-EXP) or high (NSP1 H-EXP)], as well as quantitative analyses of three independent experiments normalising the band intensities of NSP1 to the loading control GAPDH (C). Standard deviations are shown above each bar. hpi: hours pi. large NSP1 granules (Fig. 2D-F, right panel). To thoroughly investigate the association of high NSP1 expression levels with altered viroplasm structure, we analysed viroplasm morphology in NSP1-expressing cells using antibodies that recognised the three viroplasm-localised proteins NSP2, VP2 and VP6. We infected MA-104 cells with RRV and analysed the monolayers with double IF with an anti-NSP1 antibody combined with anti-VP2, anti-VP6, or anti-NSP2 antibodies at different times pi. Similar to the results shown in Fig. 2, infected cells with low NSP1 expression levels (not shown) had normal viroplasm morphology (Fig. 4A, left panel). In contrast, cells with high NSP1 expression levels frequently exhibited altered viroplasm morphology, which was characterised by large, irregular structures instead of the normal round viroplasms (Fig. 4A, right panel). The association between high NSP1 expression levels and altered viroplasm morphology was assessed at each time pi by classifying $150 \mathrm{RRV}$-infected cells according to the level of NSP1 expression (low or high) and the distribution of NSP2 (normal or altered) (Fig. 4B). This analysis indicated that more than half of the cells with high NSP1 expression levels displayed altered viroplasm morphology, suggesting that high NSP1 expression levels are associated with altered viroplasm morphology.

High NSP1 expression levels in RRV-infected cells increase in an MOI-dependent manner - Based on prior studies reporting that viral proteins and/or viral mRNAs are able to prevent the degradation of NSP1 by the proteasome (Pina-Vazquez et al. 2007), we hypothesised that high MOIs would enhance the expression level of NSP1 due to the increased accumulation of viral mRNAs and proteins under such conditions. Others have found that in RRV-infected MA-104 cells, the expression level of the major viroplasm protein NSP2 and the number and size of viroplasms increase with MOI. At 8 hpi, a 20 -fold increase in MOI (from 0.510) increased the expression level of NSP2 6-fold and the number of viroplasms 1.3-fold (Carreno-Torres et al. 2010). In contrast, silencing the expression of viral proteins that are essential for viroplasm formation, such as NSP2, greatly reduced the formation of viroplasms and the synthesis of viral mRNAs in these inclusion bodies (Silvestri et al. 2004). These data suggest that high MOIs enhance viral mRNA and protein synthesis to some extent. To investigate the effect of MOI on NSP1 expression, we infected MA-104 cells with RRV at different MOIs and semi-quantitatively analysed the expression levels of NSP1 using IF. To synchronise the infection, the cells were treated after virus adsorption with the neutralising Mab $4 \mathrm{~F} 8$ at $4^{\circ} \mathrm{C}$, which neutralises RRV virions adsorbed to the cell (Ruggeri \& Greenberg 1991). In our study, treatment with Mab 4F8 neutralised $99.9 \%$ of the infectivity of RRV pre-adsorbed to MA104 cells (data not shown). The RRV-infected MA-104 cells were synchronised and the cells were processed for IF with anti-NSP1 at 15 hpi. The NSP1-expressing cells were classified according their expression levels (low or high) and the results demonstrated that the fraction of cells with high NSP1 expression levels increased 
by approximately $27 \%$ after a 16 -fold increase in MOI from 0.5-8 (Fig. 5). These results indicate that the NSP1 expression level increases at late times after infection in an MOI-dependent manner.

\section{DISCUSSION}

Because a detailed study of NSP1 expression throughout the infection process was lacking, our first aim was to determine the intracellular distribution of NSP1 in MA-104 cells infected with RRV. NSP1 localised to the cytoplasm and formed granules that were heterogeneous in size. NSP1 preferentially localised to the perinuclear region of RRV-infected cells, in contrast to the homogenous cytoplasmic distribution of recombinant NSP1. Moreover, recombinant NSP1 was diffusely localised throughout the cytoplasm, whereas diffuse localisation was not detected in RRV-infected cells. Based on the diffuse localisation of recombinant NSP1, we cannot exclude the possibility that a fraction of NSP1 localises diffusely in the cytoplasm in RRV-infected cells below the detection level of our anti-NSP1 serum. The perinuclear localisation of NSP1 appears to be a common trait across different RVA strains, as the infection of MA-104 cells with a different viral strain, SA11, also led to the preferential localisation of NSP1 in the peri- nuclear region (Hua \& Patton 1994, Hua et al. 1994). The dependence of perinuclear distribution on viral infection suggests that viral factors are involved in determining this localisation. Viral components, such as viroplasms, localise to the perinuclear region (Silvestri et al. 2004) and the acquisition of the third protein layer of progeny virions involves budding through the ER (Estes \& Kapikian 2007). It is likely that viral components in the perinuclear region interact with NSP1, leading to its accumulation in this region.

We also determined the kinetics of the intracellular localisation of NSP1 in MA-104 cells infected with RRV. During the first $9 \mathrm{hpi}$, small NSP1 granules predominated in contrast to late times (12-18 hpi), when large granules predominated. Coincident with the emergence of large granules, a high proportion (30-40\%) of infected cells showed large areas with confluent NSP1 granules. These results suggest that the formation of large NSP1 granules and their confluence are a consequence of NSP1 abundance at late times pi. Notably, NSP1 proteins from different RVA strains differ widely in their amino acid sequences and their mechanisms to subvert the host antiviral response, which can vary in different cell types (Arnold \& Patton 2010). Therefore, the results of this study cannot be extrapolated to other viral strains or cell types.
A
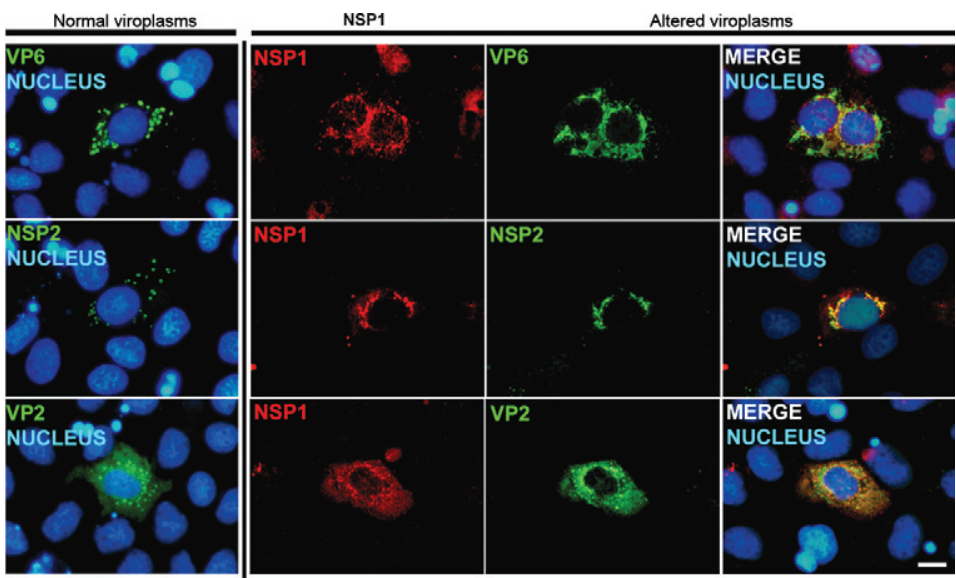

B

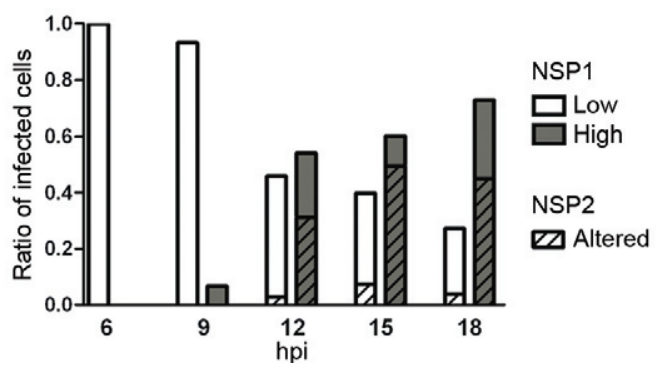

Fig. 4: high levels of non-structural protein 1 (NSP1) expression in rhesus rotavirus (RRV)-infected cells correlate with altered viroplasm morphology. MA-104 cell monolayers were infected with RRV at a multiplicity of infection of 0.1 . At $12 \mathrm{~h}$ post-infection (hpi), the cells were processed using immunofluorescence with anti-NSP1 (red) and one of three antibodies against proteins that localise in viroplasms (green): antiviral protein (VP)6, anti-NSP2 or anti-VP2. The nuclei were visualised with 4',6-diamino-2-phenylindole (blue) (A). Representative images of normal or altered viroplasm morphology are shown, as indicated above the images. The association between high levels of NSP1 expression and altered viroplasm morphology was assessed at each pi time by classifying 150 rotavirus infected cells according to the level of NSP1 expression (low or high) and the distribution of NSP2 (normal or altered) (B). Bar $=10 \mu$. 
A previous analysis of RRV replication in MA-104 cells demonstrated that the production of infectious progeny and genome replication follow similar kinetic patterns, with a small linear increase during the first $6 \mathrm{hpi}$ and a logarithmic increase from 6-12 hpi (Ayala-Breton et al. 2009). Using the same cell line, virus and MOI that Ayala-Breton et al. (2009) used to determine the kinetics of viral replication, we observed that NSP1 had a low expression level during the logarithmic increase in infectious progeny production. In contrast, NSP2 starts to accumulate sharply at $4 \mathrm{hpi}$, reaching a very high level at 8 hpi, much earlier than NSP1 (Carreno-Torres et al. 2010). These data show that NSP1 is a late viral protein in MA104 cells infected with RRV. NSP1 mRNA is approximately 1.4 times the length of NSP2 mRNA. It is unlikely that this difference would explain the different expression levels of these proteins because translation initiation is usually the rate-limiting step for protein synthesis (Mathews et al. 2007). The translational efficiency of NSP1 mRNA is slightly lower than VP6 mRNA in MA104 cells infected with B641; however, the difference is small (Mitzel et al. 2003). At 2 hpi, Mitzel et al. (2003) found $67 \%$ of NSP1 mRNA and $52 \%$ of VP6 mRNA in sub-polysomal fractions. In contrast to the mild regulatory effect of the translational efficiency of NSP1 mRNA, the expression level of NSP1 is strongly regulated posttranslationally (Pina-Vazquez et al. 2007). Pina-Vazquez et al. (2007), found that up to 11-fold of NSP1 expressed in mammalian cells was degraded by the proteasome.

It is unclear why NSP1 reaches a high expression level coincidently with the plateau of RRV replication at 12 hpi. Moreover, the high expression level of NSP1 at late times pi correlates with altered viroplasm morphology. Previous studies have found altered viroplasms in RVAinfected cells treated with a siRNA that inhibits the expression of the cellular kinase CK1 $\alpha$, leading to impaired phosphorylation of the viral protein NSP5 (Campagna et

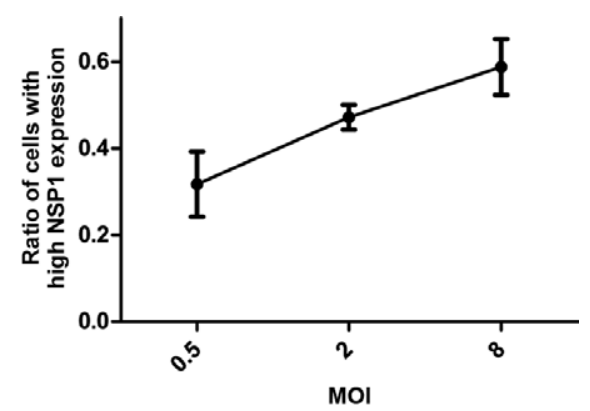

Fig. 5: high levels of non-structural proteins 1 (NSP1) expression in rhesus rotavirus (RRV)-infected cells depend on multiplicity of infection (MOI). MA-104 cells were infected with RRV at MOIs of $0.5,2$ or 8 . To synchronise the infection, the cells were washed $1 \mathrm{~h}$ post-infection (hpi) and treated with the neutralising Mab $4 \mathrm{~F} 8$ for $1 \mathrm{~h}$ at $4^{\circ} \mathrm{C}$, as previously described, to neutralise the input virus (Ruggeri \& Greenberg 1991). At $15 \mathrm{hpi}$, the cells were processed using immunofluorescence with anti-NSP1 and the NSP1-expressing cells were classified according to the NSP1 expression level (low or high). The ratio of cells with high levels of NSP1 expression relative to all cells expressing NSP1 is shown. Standard deviations are shown for each MOI. al. 2007). Under these conditions, viroplasms adopt irregular shapes resembling aggregations of smaller viroplasms. It is unclear whether there is any relationship between the altered viroplasms in cells treated with siRNA against CK1 $\alpha$ and the altered viroplasms described in this study. Further study will be needed to determine whether the decrease in viral replication altered viroplasm morphology and high NSP1 expression are somehow related.

In this study, we determined the effect of MOI on NSP1 expression. Our results demonstrate that the fraction of cells with elevated NSP1 levels increased proportionally with the MOI. We hypothesised that high MOIs enhanced NSP1 expression levels due to the increased accumulation of viral mRNAs and proteins, which are known to stabilise NSP1 against proteasomal degradation. Our experimental setup included late time points up to 18 hpi. From 12-18 hpi, considerable numbers of infectious viruses can be released to initiate a second round of infection. Therefore, we cannot exclude the possibility that secondary infections contributed to the increased expression of viral components that stabilise NSP1 against proteasomal degradation. Moreover, other factors may be involved in enhancing NSP1 expression at high MOIs. High MOIs of the polyomavirus SV40 favour the production of defective interfering (DI) particles, which can replicate only in the presence of a helper virus, but interfere with such replication (Huang \& Baltimore 1970). At high MOIs, the probability that a DI particle and a non-defective virus co-infect a given cell increases. Because DI particles affect the replication of their helper viruses, the elevated levels of NSP1 and the altered viroplasm morphology observed with increased MOI may be consequences of defects in rotavirus replication caused by DI particles. Indeed, the production of DI particles in RVA stocks may be favoured by propagation of the virus at high MOIs, a common practice in RVA propagation (Gray \& Desselberger 2000) due to the difficulty of obtaining high titres at low MOIs.

A recent study proposed that NSP1 plays a role in regulating RVA gene expression (Chung \& McCrae 2011). Chung and McCrae proposed that at late times pi, the binding of NSP1 to viral mRNA favours the packaging and replication of the viral genome. This model is consistent with our findings that NSP1 localises close to viroplasms in the perinuclear region and that NSP1 expression kinetics change late in the infection.

In this study, we found that NSP1 localises preferentially to the perinuclear region in the cytoplasm of RRVinfected MA-104 cells. We also found a late shift from low to high expression levels; therefore, NSP1 can be considered a late viral protein in MA-104 cells infected with RRV. Combined with prior studies on the susceptibility of NSP1 to proteasomal degradation, our results suggest that the late shift from low to high NSP1 expression levels occurs as a result of altered NSP1 turnover.

\section{ACKNOWLEDGEMENTS}

To HB Greenberg, for providing the Mabs used in this study, to B Moss, for providing the plasmid p.VOTE.1 and the vaccinia virus vT7lacOI that were used in the VOTE expression system, and to R León-Rodríguez, for technical assistance. 


\section{REFERENCES}

Anonymous 2008. Rotavirus surveillance - worldwide, 2001-2008. MMWR Morb Mortal Wkly Rep 57: 1255-1257.

Arnold MM, Patton JT 2010. Diversity of interferon antagonist activities mediated by NSP1 proteins of different rotavirus strains. J Virol 85: 1970-1979.

Ayala-Breton C, Arias M, Espinosa R, Romero P, Arias CF, Lopez S 2009. Analysis of the kinetics of transcription and replication of the rotavirus genome by RNA interference. J Virol 83: 8819-8831.

Bass DM, Mackow ER, Greenberg HB 1990. NS35 and not vp7 is the soluble rotavirus protein which binds to target cells. $J$ Virol 64: 322-330.

Cabral-Romero C, Padilla-Noriega L 2006. Association of rotavirus viroplasms with microtubules through NSP2 and NSP5. Mem Inst Oswaldo Cruz 101: 603-611.

Campagna M, Budini M, Arnoldi F, Desselberger U, Allende JE, Burrone OR 2007. Impaired hyperphosphorylation of rotavirus NSP5 in cells depleted of casein kinase lalpha is associated with the formation of viroplasms with altered morphology and a moderate decrease in virus replication. J Gen Virol 88: 2800-2810.

Carreno-Torres JJ, Gutierrez M, Arias CF, Lopez S, Isa P 2010. Characterization of viroplasm formation during the early stages of rotavirus infection. Virol $J$ 7: 350 .

Chung KT, McCrae MA 2011. Regulation of gene expression by the NSP1 and NSP3 non-structural proteins of rotavirus. Arch Virol 156: 2197-2203.

Coria-Galindo E, Rangel-Huerta E, Verdugo-Rodriguez A, Brousset D, Salazar S, Padilla-Noriega L 2009. Rotavirus infections in Galapagos Sea lions. J Wildl Dis 45: 722-728.

Earl PL, Americo JL, Wyatt LS, Eller LA, Montefiori DC, Byrum R, Piatak M, Lifson JD, Amara RR, Robinson HL, Huggins JW, Moss B 2007. Recombinant modified vaccinia virus Ankara provides durable protection against disease caused by an immunodeficiency virus as well as long-term immunity to an orthopoxvirus in a non-human primate. Virology 366: 84-97.

Elroy-Stein O, Moss B 2007. Gene expression using the vaccinia/T7 RNA polymerase hybrid system. In F Ausubel, R Brent, R Kingston, D Moore, J Seidman, J Smith, K Struhl, Current protocols in molecular biology, John Wiley and Sons, New York, p. 16.19.11.

Ericson BL, Graham DY, Mason BB, Estes MK 1982. Identification, synthesis and modifications of simian rotavirus SA11 polypeptides in infected cells. $J$ Virol 42: 825-839.

Estes M, Kapikian A 2007. Rotaviruses. In D Knipe, P Howley, Fields virology, Vol. 2, 5th ed., Lippincott Williams and Wilkins, Philadelphia, p. 1747-1786.

Estes MK, Graham DY, Gerba CP, Smith EM 1979. Simian rotavirus SA11 replication in cell cultures. $J$ Virol 31: 810-815.

Graff JW, Ewen J, Ettayebi K, Hardy ME 2007. Zinc-binding domain of rotavirus NSP1 is required for proteasome-dependent degradation of IRF3 and autoregulatory NSP1 stability. J Gen Virol 88: 613-620.
Gray J, Desselberger U 2000. Rotavirus: methods \& protocols, Humana Press, Totowa, $262 \mathrm{pp}$

Hua J, Chen X, Patton JT 1994. Deletion mapping of the rotavirus metalloprotein NS53 (NSP1): the conserved cysteine-rich region is essential for virus-specific RNA binding. $J$ Virol 68 : 3990-4000.

Hua J, Patton JT 1994. The carboxyl-half of the rotavirus nonstructural protein NS53 (NSP1) is not required for virus replication. Virology 198: 567-576.

Huang AS, Baltimore D 1970. Defective viral particles and viral disease processes. Nature 226: 325-327.

Johnson MA, McCrae MA 1989. Molecular biology of rotaviruses. VIII. Quantitative analysis of regulation of gene expression during virus replication. J Virol 63: 2048-2055.

Kaljot KT, Shaw RD, Rubin DH, Greenberg HB 1988. Infectious rotavirus enters cells by direct cell membrane penetration, not by endocytosis. J Virol 62: 1136-1144.

Laemmli UK 1970. Cleavage of structural proteins during the assembly of the head of bacteriophage T4. Nature 227: 680-685.

Mathews MB, Sonenberg N, Hershey JWB 2007. Origins and principles of translational control. In MB Mathews, N Sobenberg, JWB Hershey, Translational control in biology and medicine, CSHL Press, Cold Spring Harbor, p. 1-40.

McCrae MA, Faulkner-Valle GP 1981. Molecular biology of rotaviruses. I. Characterization of basic growth parameters and pattern of macromolecular synthesis. J Virol 39: 490-496.

Mitzel DN, Weisend CM, White MW, Hardy ME 2003. Translational regulation of rotavirus gene expression. J Gen Virol 84: 383-391.

Padilla-Noriega L, Paniagua O, Guzman-Leon S 2002. Rotavirus protein NSP3 shuts off host cell protein synthesis. Virology 298: 1-7.

Pina-Vazquez C, de Nova-Ocampo M, Guzman-Leon S, PadillaNoriega L 2007. Post-translational regulation of rotavirus protein NSP1 expression in mammalian cells. Arch Virol 152: 345-368.

Ruggeri FM, Greenberg HB 1991. Antibodies to the trypsin cleavage peptide VP8 neutralize rotavirus by inhibiting binding of virions to target cells in culture. $J$ Virol 65: 2211-2219.

Shaw RD, Stoner-Ma DL, Estes MK, Greenberg HB 1985. Specific enzyme-linked immunoassay for rotavirus serotypes 1 and 3 . J Clin Microbiol 22: 286-291.

Silvestri LS, Taraporewala ZF, Patton JT 2004. Rotavirus replication: plus-sense templates for double-stranded RNA synthesis are made in viroplasms. J Virol 78: 7763-7774.

Svensson L, Padilla-Noriega L, Taniguchi K, Greenberg HB 1990. Lack of co-segregation of the subgroup II antigens on genes 2 and 6 in porcine rotaviruses. $J$ Virol 64: 411-413.

Ward GA, Stover CK, Moss B, Fuerst TR 1995. Stringent chemical and thermal regulation of recombinant gene expression by vaccinia virus vectors in mammalian cells. Proc Natl Acad Sci USA 92: 6773-6777. 